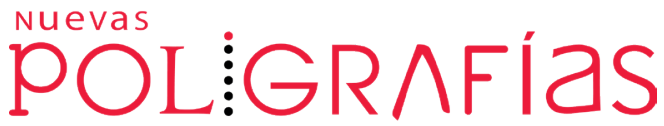

Revista de teoría literaria y literatura comparada
NÚMERO 5 | febrero - julio 2022 | 59-76

DOI: 10.22201/ffyl.nuevaspoligrafias.2022.5.1552

Recibido: 15-08-2021 | Aceptado: 22-10-2021

\title{
El diÁlogo ENTRE LOS OPUESTOS POR MEDIO DE LOS PERSONAJES EN A Tale of Two Cities de Charles Dickens
}

\author{
The Dialogue Between Opposites Through the Characters in \\ Charles Dickens's a Tale of Two Cities
}

\author{
Lia GALVÁN LISKER \\ Facultad de Filosofía y Letras ${ }^{1}$ \\ Universidad Nacional Autónoma de México | México \\ Contacto: lia.galvanlisker@gmail.com
}

\begin{abstract}
Resumen
Este artículo busca analizar, por medio de las estrategias de caracterización explicadas por Luz Aurora Pimentel, la transformación de ciertos pares de personajes en la conocida novela histórica de Charles Dickens A Tale of Two Cities (1859). En un inicio los pares de personajes de Alexander Manette y Ernest Defarge, Jarvis Lorry y Jerry Cruncher, y Charles Darney y Sidney Carton son retratados de manera que contrastan entre sí tanto de manera física como moral. Al inicio de la novela, Defarge, Cruncher y Carton comparten características como desapego emocional, deshonestidad y crueldad en distintos grados, mientras que Manette, Lorry y Darney son caracterizados como compasivos, honestos y solidarios. Sin embargo, a lo largo de la novela todos estos personajes revelan a través de sus acciones y discurso figural distintas maneras en las que llegan a ser similares a su aparente "contraparte", pues para el final de ésta todos ellos comparten características con su par por medio de un proceso de convivencia y aprendizaje. Arguyo que el hecho de que se dé la posibilidad de una reformulación en la identidad de los personajes demuestra la existencia de una escala
\end{abstract}

\begin{abstract}
This article seeks to analyze, through the characterization strategies explained by Luz Aurora Pimentel, the transformative process of certain pairs of characters in Charles Dickens' well-known historical novel A Tale of Two Cities (1859). At the beginning of the narration, the pairs of characters of Alexander Manette and Ernest Defarge, Jarvis Lorry and Jerry Cruncher, and Charles Darney and Sidney Carton are portrayed in a contrasting manner regarding both their physical and moral attributes. At the beginning of the novel, Defarge, Cruncher, and Carton share characteristics such as emotional detachment, dishonesty, and recklessness in different degrees, while Manette, Lorry, and Darney are characterized as compassionate, honest, and solidary. However, throughout the novel, all of these characters reveal, through their figural speech and their actions, different ways in which they become similar to their apparent "counterpart," for, by the end of it, all of them share characteristics with their double through a process of coexistence and learning. I argue that the fact that there is a possibility of reformulation in the characters' identities demonstrates the existence of nuances
\end{abstract}

1 Estudiante de la Licenciatura en Lengua y Literaturas Modernas (Letras Inglesas) 
de grises entre los extremos morales representados en la novela, los cuales en un inicio aparentan ser inamovibles; esto, asimismo, desestabiliza concepciones como "héroe" y "villano", al igual que las supuestas diferencias entre los personajes de ambos bandos.

Palabras clave: A Tale of Two Cities; Charles Dickens; novela histórica; caracterización; identidad, dobles between the moral extremes represented in the novel which at the beginning appear to be immovable. This, at the same time, destabilizes conceptions such as "hero" and "villain" as well as the supposed differences between the characters from both sides.

Keywords: A Tale of Two Cities; Charles Dickens; historical novel; characterization; identity; doubles

Tale of Two Cities, la novela histórica que Charles Dickens publicó en 1859,
cuenta, por un lado, la situación social en Francia, particularmente la histo-
ria del matrimonio de los Defarge y, por el otro, la de la familia Manette y sus allegados en Inglaterra. La novela se desarrolla desde los años previos a la Revolución Francesa hasta llegar al Gran Terror, y relata cómo estos eventos cambian las vidas de los personajes por completo. La Revolución Francesa fue un período bastante conocido por los victorianos así que, al retratar ésta en su novela, Dickens no pretendía "to illuminate an obscure corner of history, but rather to retell a familiar story" (Davis, 2008: 413), lo cual hace al enfocarse en la historia de individuos anónimos más que en personajes y eventos históricos famosos.

Desde el título, A Tale of Two Cities, queda sugerido que Dickens va a explorar dualidades o, como Albert D. Hutter (1978) establece, "the 'twoness' of everything to follow" (455). Esto queda aún más claro con la primera, y bastante conocida, oración de la novela: "It was the best of times, it was the worst of times..." (Dickens, 1981: 3). A lo largo del primer capítulo, el cual describe el periodo en el que se sitúa la novela, Dickens utiliza una retórica basada en contrastes. Devin Griffiths (2013) establece que "the alternating statements reach out to delimit, in their absolute contradiction, the widest compass of opposed opinions" (812). Sin embargo, aunque a primera vista pareciera ser que Dickens está estableciendo el mundo de la novela entre opuestos claramente definidos, a lo largo de la misma los logra representar de forma que demuestra las maneras en las que puede haber similitudes entre éstos. De esta forma, "the use of doubles may [also] suggest, not splitting, but reunifying 
something once divided or divisible" (Glancy, 2006: 86), lo cual se refleja por medio de las diferencias y similitudes retratadas entre Francia e Inglaterra, los tiempos históricos y los personajes.

El objetivo de este artículo será explorar la forma en la que Charles Dickens representa la dualidad de manera ambivalente en A Tale of Two Cities, en particular, a través de la caracterización de ciertos pares de personajes. Al estudiar las obras de Dickens, los autores Gillman y Patten (1985) propusieron la categoría "tropological doubles", la cual se refiere a "the pairs of characters that act out moral polarities" (442), es decir, los personajes que representan los extremos opuestos de la moralidad. Estos tipos de personajes "are not merely different but contradictory [...] to others toward whom they are overtly antagonist" (Rosenberg, 1992: 158). En A Tale of Two Cities hay tres pares de personajes que en un inicio parecen funcionar como "tropological pairs" al ser retratados como opuestos, pero que a lo largo de la novela demuestran no serlo ya que a través de su discurso figural y sus acciones revelan todas las maneras en las que llegan a ser similares. El análisis de estos personajes se sustentará en las estrategias de caracterización explicadas por Luz Aurora Pimentel y la finalidad de éste será entender cómo la posibilidad de una reformulación en la identidad de los personajes demuestra la existencia de una escala de grises entre los extremos morales representados en la novela.

Al tratar la moralidad en su novela, Dickens estaba retomando un tema importante para la época, pues "Victorian England is much noted for its strong attachment to moral concern" (Xiao, 2015: 1815). En la era victoriana, la moralidad era concebida de forma que estaba entretejida con un estricto código de modales los cuales aseguraban estándares de "respetabilidad". Estos estándares, de acuerdo a William A. Madden (1961: 96), inculcaban valores como la prudencia, diligencia y templanza, y funcionaban, asimismo, como instrumentos de control social mediante los cuales la clase media buscaba dominar a la clase trabajadora. Así, la noción de respetabilidad estaba asociada, sobre todo, con la clase media para después extenderse al resto de las clases. Ya que éste era el estándar mediante el cual se juzgaba la moralidad de una persona, los victorianos le daban un gran valor a mantener la apariencia y los modales de una buena conducta (Madden, 1961: 92). Con respecto a esto, Dickens comentó que "he was tired of making his heroes conform to Victorian standards of respectability, standards that he felt were hypocritical, unrealistic, and limiting to art" (Glancy, 2006: 16). Esta postura es significativa pues, tradicionalmente, "the realist novel largely accepts middle-class ethics and mores" (Shires, 2001: 25), a lo 
cual Dickens se resiste en cierto grado a través de la caracterización de sus personajes, pues les provee de rasgos que van más allá de los estándares de respetabilidad y que, a su vez, cuestionan la manera en la que se definían los extremos morales al igual que categorías como "héroes" y "villanos".

El primer par de personajes que será analizado son Alexandre Manette, el doctor y ex prisionero de la Bastilla, y Ernest Defarge, el dueño de una tienda de vinos y líder revolucionario. Estos personajes se relacionan ya que Defarge solía trabajar para Manette y, cuando éste es rescatado de la Bastilla, Defarge es quien le da asilo en su tienda. El segundo par es Jarvis Lorry y Jerry Cruncher, los cuales son los primeros en aparecer en la novela, y están conectados puesto que ambos trabajan en el Banco de Tellson. Por último están Charles Darnay y Sidney Carton, quienes están vinculados ya que, aunque no se conocen, Carton salva a Darnay de ser juzgado erróneamente por cargos de espionaje gracias a que son muy similares en su aspecto físico. Así, la vida y el destino de estos dos personajes quedan enlazados por el resto de la novela.

El análisis llevado a cabo estará centrado en la caracterización de estos tres pares de personajes. Luz Aurora Pimentel (1998) define caracterización, por medio de la teoría de Barthes, como "la 'imagen' sintética de la apariencia física de los personajes así como su retrato moral a través de un sinnúmero de detalles [...] y los rasgos de personalidad correspondientes" (61) - es decir, la forma en la que el autor construye la identidad, tanto física como moral, de un personaje de manera que su personalidad sea reconocible en la trama-. Pimentel explica varias estrategias de caracterización, entre ellas el retrato y el discurso figural. El retrato se trata de la descripción de la identidad física de un personaje la cual puede provenir del narrador o del discurso de otros personajes (71). Pimentel explica cómo por medio de la descripción física también se da el retrato moral de un mismo personaje (75). El discurso figural se refiere al discurso de los personajes, el cual puede ser enunciado de forma dramática $o$ narrativa. A través de éste "se marcan asimismo los grados de transformación de un personaje" (88); es decir, funciona como una manera de trazar el cambio en la identidad de los personajes.

Es importante tener en mente que la forma de conceptualizar la identidad ha cambiado a lo largo del tiempo. De acuerdo con Linda Shires (2001), a partir de la mitad del siglo xix y en adelante, los textos literarios victorianos comienzan a demostrar "a profound discomfort with a central 'I' of unmediated experience, a coherent self" (69). Esta desestabilización del "yo" contrasta con las novelas realistas características de este periodo (categoría en la que suelen encontrarse las novelas de Dickens) 
pues éstas enfatizan un modelo de coherencia o consistencia no sólo en su forma sino también en la construcción de los personajes (Shires, 2001: 63). Si bien esta noción puede relacionarse con A Tale of Two Cities, también es importante tomar en cuenta que "no es indiferente que el origen de la información sobre el ser y el hacer de un personaje y su valoración provengan del discurso del narrador, del de otros personajes o del propio personaje" (Pimentel, 1998: 69). En este caso, busco hacer una distinción entre la información provista por la voz narrativa que establece retratos iniciales coherentes y consistentes y el discurso figural de los personajes, el cual funciona para señalar las transformaciones identitarias de los personajes a través de su propia voz. Si bien los personajes al principio de la novela son caracterizados por la voz narrativa de una manera firme, también llevan procesos identitarios complejos en donde se transforman al convivir con sus aparentes "dobles", lo cual refleja que las identidades no pueden sostenerse sin la cooperación de otros (Fludernik, 2007: 261). De esta forma, la identidad en la novela puede entenderse como "una descripción discursiva emocionalmente cargada de nosotros mismos que está sujeta al cambio" (Golubov, 2015: 140). Esta noción se alinea con las preocupaciones con respecto a la conceptualización de la identidad a partir de la segunda mitad del siglo xix como un elemento inestable y, por lo tanto, propenso a transformarse.

Alexandre Manette y Ernest Defarge son la primera pareja de personajes que aparentan funcionar como opuestos. La voz narrativa establece de manera clara el contraste entre ambos mediante sus retratos iniciales. Ambos son introducidos en el quinto capítulo. El primero en ser retratado por la voz narrativa es Ernest Defarge:

This wine-shop keeper was a bull-necked, martial-looking man of thirty, and he should have been of a hot temperament [...]. Neither did he wear anything more on his head than his crisply-curling short dark hair [...] [he had] good eyes [...]. Good-humored looking on the whole, but implacable looking too; evidently a man of a strong resolution and set purpose. (Dickens, 1981: 32)

La voz narrativa empieza por caracterizar a Defarge utilizando adjetivos que demuestran su fuerza tanto física, al hacer referencia a un toro, como mental, al utilizar el adjetivo "martial" que remite a estrategias y disciplina. Después describe su temperamento como "hot", lo cual lo retrata como alguien que pone sus emociones por encima de lo racional. Esto es significativo, pues va en contra de "the bourgeois ideal of the rational man" (Shires, 2001: 65) presente en la época victoriana. Al final men- 
ciona una de sus características más importantes al describirlo como "implacable". De esta forma, su primer retrato marca la forma en la que Monsieur Defarge va a ser caracterizado por la voz narrativa en gran parte de la novela: un hombre fuerte, inteligente, apasionado y determinado por completo a la causa revolucionaria quien no entra dentro de las características "respetables" de la época victoriana.

Unas cuantas páginas después el narrador da el primer retrato de Alexandre Manette, el cual consiste en una descripción simple: "a white haired man sat on a low bench, stooping forward and very busy making shoes" (Dickens, 1981: 39). Más adelante el narrador agrega otras descripciones de las características de Manette, pero éstas se dan de manera discontinua: "very faint voice", "haggard eyes", "pitiable and dreadful" (Dickens, 1981: 39). Por medio de este retrato se establece un contraste directo entre las características físicas de ambos personajes, ya que mientras que Defarge tiene el cabello café y "good eyes", Manette tiene el cabello blanco y "haggard eyes". También es significativo que Manette demuestre valores típicos de la era victoriana como "hard work" (Madden, 1961: 98) y "self discipline" (1961: 99), lo cual lo posiciona dentro de los estándares morales de la época. El resto de los adjetivos que el narrador usa para describir a Manette, como "pitiable" y "dreadful", lo caracterizan como un ser frágil, lo cual contrasta con la fuerza de Defarge. Incluso el hecho de que el retrato de Defarge sea un párrafo bien consolidado mientras que el de Manette se da por medio de oraciones fragmentadas refleja que Manette es visto como alguien con poca estabilidad mental y física, mientras que Defarge es fuerte en ambos aspectos.

A medida que se desarrolla la novela, Manette supera poco a poco sus traumas y se empieza a reconocer a sí mismo como la persona que era antes de su encarcelamiento. La voz narrativa hace referencia a esto al inicio del segundo libro al decir: "It would have been difficult by a far brighter light to recognize in Doctor Manette, intellectual of face and upbright of bearing, the shoemaker of the garret in Paris" (Dickens, 1981: 80). Sin embargo, sigue siendo caracterizado como un personaje atormentado, lo cual se aprecia cuando la voz narrative, después de esta primera descripción, agrega: "yet, no one could have looked at him twice, without looking again [...] the mournful cadence of his low grave voice, and to the abstraction that overclouded him fitfully" (Dickens, 1981: 80). Esto demuestra que aunque Manette está sanando, pareciera que la voz narrativa consistentemente lo retrata como marcado por su pasado, lo cual se refleja en aspectos físicos como el tono de su voz y también en los ataques de regresión que tiene a lo largo de la novela donde olvida quién es y se pone a hacer zapatos. 
A pesar de estos traumas, para el final de la novela, Manette adquiere una de las principales características de Defarge - su fuerza - lo cual se expresa mediante el discurso figural de Manette. En la tercera parte de la novela, Charles Darnay, el esposo de la hija de Manette, es encarcelado en París al tratar de salvar a alguien que fue su sirviente. En este contexto, el hecho de ser un exprisionero de la Bastilla, lo cual en toda la novela había sido un motivo de trauma y fragilidad, le da a Manette una gran influencia que él aprovecha para intentar salvar a Darnay. Él mismo dice: "my old pain has given me a power that has brought us through the barrier, and gained us news of Charles there [...] I knew I could help Charles out of danger" (Dickens, 1981: 265). El hecho de que en este diálogo utilice los pronombres personales "I" y "me" hace referencia a que sabe que él mismo es quien cuenta con poder, demostrando que es consciente del cambio en su interior y la nueva fuerza que posee. Así, a través de su propio discurso figural, Manette refleja el cambio en sí mismo donde pasó de ser retratado como una persona atormentada por su trauma a alguien que cuenta con la fuerza de voluntad para utilizarlo de una manera ventajosa, lo cual desafía la caracterización que la voz narrativa le había dado hasta el momento.

Defarge también experimenta un cambio en su personalidad para el final de la novela, el cual refleja, asimismo, a través de su discurso figural. A lo largo de la trama, Defarge manifiesta los aspectos que lo caracterizaban en su primer retrato donde la voz narrativa se refiere a él como "a man not desirable to be met in a narrow path for nothing will turn the man" (Dickens, 1981: 32), lo cual se ve en los pasajes donde, junto con su esposa, guía la revolución. Sin embargo, en la parte final de la novela, cuando Madame Defarge quiere sacrificar al resto de la familia Manette, él dice: "well, well, but one must stop somewhere [...] this doctor has suffered much, you have seen him today, you have observed his face when the paper was read the anguish of his daughter, which must be a dreadful anguish to him" (Dickens, 1981: 344). Este diálogo implica que Defarge se ha transformado desde su primer retrato ya que ha descubierto su límite y sabe que debería ser universal al utilizar el pronombre impersonal "one must" mientras que al usar palabras como "suffered" and "anguish" evidencia su capacidad para entender la profundidad de las emociones tanto de Manette como de su hija y que éstas le importan.

Monsieur Defarge, de esta manera, demuestra ser compasivo, un rasgo que el Doctor Manette ha ejercido también a lo largo de la novela, en particular en la carta donde revela su conexión con los Evrémonde en donde se preocupa de forma genuina por su paciente (Dickens, 1981: 328). Así, tanto Defarge como Manette, demues- 
tran a través de su discurso ser fuertes y compasivos para el final de la novela aunque ambas características no pertenezcan e incluso contradigan sus retratos iniciales. Es significativo que este cambio se marque a través del discurso figural de ambos personajes, pues, como explica Pimentel (1998), "es evidente que todo discurso figural [...] constituye un punto de vista sobre el mundo; una postura ideológica" (86). Así, este cambio en ambos, pero en particular en Defarge, revela que existe una transformación en su postura ideológica que le permite comenzar a reformularse de manera positiva, no en el sentido de los estándares de respetabilidad de la época, sino en relación con una moralidad en donde la compasión juega una parte fundamental.

De una manera similar, Jarvis Lorry y Jerry Cruncher, los primeros personajes importantes en aparecer en la novela, terminan por compartir características para el final de ésta pese a que, en un principio, sean retratados como opuestos. El narrador da primero un retrato exhaustivo de Lorry en el cuarto capítulo, describiéndolo con los adjetivos "orderly", "methodical", "little vain", "composed" y "reserved" (Dickens, 1981: 17), los cuales lo caracterizan como un hombre pulcro, ordenado y profesional. Además de esto, el narrador se enfoca en el aspecto físico de Lorry y, en particular, en la forma en la que está vestido. El narrador dice que Lorry "wore an odd little sleek crisp flaxen wig [...] which looked far more as though it were spun from filaments of silk and grass. His linen [...] was as white as the top of the waves..." (Dickens, 1981: 17). Esto refleja por un lado su carácter "suave" al comparar su vestimenta con la seda y la espuma de las olas y, por el otro, completa su aspecto como un caballero respetable para la época. Lorry es, por lo tanto, uno de los personajes que más encarna los estándares de respetabilidad tales como "sobriety, frugality, foresight" (Madden, 1961: 98), lo cual lo posiciona cerca de los estándares morales convencionalmente positivos para la época.

El retrato de Jerry Cruncher que la voz narrativa provee en el capítulo irónicamente titulado "the honest trademan", contrasta por completo con el de Mr. Lorry. La primera vez que la voz narrativa lo menciona en el capítulo, se refiere a él como un "odd-job-man" que, según el diccionario de Cambridge, es una persona que hace varios tipos de trabajos. Sin embargo, no es casualidad que el primer adjetivo para describir a Cruncher sea "odd" ya que esto va a resonar en su personalidad. Acto seguido, se describe la casa de Cruncher donde éste está despertando y las primeras características físicas en enunciarse son su "spiky hair" y "a voice of dire exasperation" (Dickens, 1981: 56). Estos dos atributos contrastan con el retrato de Lorry donde el narrador hizo un énfasis en su blanca y ordenada peluca y por el hecho de que durante 
este pasaje no habla, ya que es descrito como reservado. El retrato de Cruncher se completa por el hecho de que, después de levantarse, lo primero que hace es lanzarle un zapato, que está sucio, a su esposa (Dickens, 1981: 56). De esta manera, Jerry Cruncher es caracterizado como un hombre mal educado, violento y desordenado y que de ninguna forma está cerca de los estándares de respetabilidad de la época victoriana.

Por medio de su primer retrato, Lorry es descrito como un hombre muy profesional y dedicado a los negocios. Esto se refleja en la primera conversación que tiene con Lucie Manette, a quien Lorry advierte que "all the relations I hold with my fellow-creatures are mere business" (Dickens, 1981: 23), en donde su discurso figural confirma el retrato inicial dado por la voz narrativa. Y ahonda en esto al decir que en estas relaciones "there is no friendship [...], no particular interest, nothing like sentiment" (Dickens, 1981: 23). Sin embargo, la voz narrativa remarca que Lorry no es como un hombre de negocios tradicional para la época, ya que demuestra tener las características de "honesty, self-denial, justice, and truthfulness". Al distinguir a Lorry de los hombres de negocios convencionales por sus rasgos positivos, Dickens critica los estándares que guiaban este tipo de instituciones y, al mismo tiempo, propone las características que deberían guiarlas en su lugar.

Lorry, asimismo, demuestra su capacidad de cambiar y evolucionar, pues, a pesar de predicar lo contrario, a lo largo de la novela se convierte en un gran y leal amigo de los Manette. Esto queda claro cuando Carton lo describe en su visión del futuro como "the good old man, so long their friend, in ten years time enriching them with all he has" (Dickens, 1981: 381). Aquí, es importante tener en cuenta que las acciones de un personaje también forman parte de su discurso figural, pues como explica Pimentel (1998), "dado que es el lenguaje el sistema de significación por medio del cual se proyecta ese mundo narrado, tanto los acontecimientos verbales como los no verbales se transmiten por ese medio" (83). De esta manera, las acciones de Lorry indican su potencial de transformación donde, a pesar de contradecir su retrato inicial, él es capaz de desarrollar relaciones significativas. Así, además de ser caracterizado como un hombre honesto y dedicado, es sobre todo esta última capacidad lo que posiciona a Lorry cerca del extremo moral positivo, pues, de acuerdo con Carl Dennis (1969), "for Dickens, the source of moral life and the possibility of moral action [...] is rooted in the emotions" (1242), lo cual Lorry encarna al dejar de lado, en ciertos sentidos, al hombre de negocios racional para arriesgar su estilo de vida por los Manette, convirtiéndose en su amigo cercano en el proceso. 
En comparación, se puede decir que el retrato moral de Cruncher se consolida cuando se revela su segunda profesión como "resurrection man", es decir, alguien que desentierra cadáveres para venderlos. La connotación moral de esto es aún más significativa cuando la revelación de que éste es el otro trabajo de Cruncher se da por medio de su hijo. Él relata que "they were still fishing perseveringly, when he peeped in the gate for the second time; but, now they seemed to have got a bite [...] Young Jerry very well knew what it would be; but, when he saw it, and saw his honoured parent about to wrench it open, he was so frightened, being new to the site, that he made off again..." (Dickens, 1981: 163). El adjetivo "honoured", que era la visión que el hijo tenía de su padre, contrasta con el hecho de que está abriendo un ataúd. La corrupción de la inocencia del hijo, la cual se reflejaba en el hecho de que describe esto como una escena de pesca, demuestra lo negativo que es, en términos morales, lo que Jerry Cruncher hace como segundo trabajo.

Hacia el final de la novela, sin embargo, Cruncher está a la altura del nombre del capítulo donde es descrito por primera vez y con mucho esfuerzo se convierte en "the honest trademan". Esto sucede cuando revelar la información correspondiente a su segundo trabajo se convierte en un momento vital para que Sidney Carton pueda llevar a cabo su plan de salvar a los Manette. Después de algunos minutos donde Cruncher duda si revelar la información, al saber lo que esto implica, dice: "you buried pavingstones and earth in that coffin. Don't go and tell me that you buried Cly, it was a take in" (Dickens, 1981: 309). En este diálogo, Cruncher, que por lo general habla de manera desordenada y difícil de entender, se muestra claro y autoritativo. Esto es significativo pues "un personaje no sólo se caracteriza por sus actos sino por las peculiaridades de su discurso; en él se observan los rasgos idiolectales, estilísticos e ideológicos —entre otros- que contribuyen a la construcción gradual de una personalidad" (Pimentel, 1998: 88). Así, en este caso, que sea autoritativo en su manera de hablar no sólo le da la fuerza necesaria a esta afirmación para que sea útil para el plan de Carton, sino que también demuestra el inicio de una transformación en la construcción identitaria de Cruncher. Sin embargo, y como ya lo sabía Cruncher, la pregunta "how do you know it?” (Dickens, 1981: 309) resulta inevitable después de su declaración, donde una vez más evita decir la verdad. Esto implica que en el momento no está todavía dispuesto del todo a cambiar ni a dejar sus viejos hábitos.

Es hasta que Mr. Lorry confronta directamente a Cruncher que hay un cambio significativo en su persona. Lorry primero le dice, "my mind misgives me much that you have used the respectable and great house of Tellson as a blind, and that you 
have had an unlawful occupation of an infamous description" (Dickens, 1981: 311), lo cual muestra una vez más el sentido de lo correcto y lo moral de Lorry al utilizar "unlawful" y "infamous" para describir esta actividad. Acto seguido, Lorry agrega, "It may be that I shall yet stand your friend, if you deserve it, and repent in actions..." (Dickens, 1981: 313), en donde también demuestra que ha sido capaz de desarrollar una relación de amistad con Cruncher incluso aunque fueran posicionados como opuestos en un principio. Es debido a este ultimátum y a la confrontación de su supuesta contraparte que Cruncher cae en cuenta de la gravedad que implica lo que solía hacer para ganar dinero. Cruncher, al reflexionar sobre sus acciones para el final de la novela, declara a través de su discurso figural "with an ashy and solemn visage 'them poor things well out o' this, never no more will I do it, never no more!'” (Dickens, 1981: 369). Sin embargo, el hecho de que esto no se lo dice a Lorry sino a Miss Pross demuestra que esta declaración no se debe a que quiera conservar la amistad de Lorry y su trabajo, sino que refleja un cambio profundo en su persona. Por esto mismo, las "poor things" a las que se refiere no son sólo el acto de desenterrar cuerpos, sino también el de ser violento con su esposa y maleducado en general. Así, gracias a su propia honestidad y a la influencia de Lorry, él afirma su voluntad de cambiar y mejorar su comportamiento. De esta manera, para el final de la novela, aunque al principio son retratados como completos opuestos, tanto Lorry como Cruncher demuestran a través de su discurso y sus acciones ser honestos y capaces de cambiar -Lorry al desarrollar relaciones fuera de los negocios y Cruncher al estar dispuesto a dejar de ser el hombre que solía ser.

La pareja de dobles que son caracterizados con diferencias morales que aparentan ser más acentuadas son Sydney Carton y Charles Darnay, ya que, aunque están unidos por su parecido físico, en un inicio ésta es la única similitud que parecen compartir. Las diferencias en sus personalidades son enfatizadas por la voz narrativa a través de sus retratos. Darnay, en su primera aparición, es descrito por el narrador como: "a young man of five-and twenty, well-grown and well-looking [...] His condition was that of a young gentleman [...] His hair, which was long and dark, was gathered in a ribbon at the back of his neck [...] he was quite self- possessed" (Dickens, 1981: 63). En otras palabras, es caracterizado como un caballero, bien arreglado y con buenos modales, y posicionado dentro de los estándares de respetabilidad de la época. Por su parte, Carton, que es retratado unas páginas más tarde, es descrito de manera que parece ser lo contrario por completo: "This one man, with his torn gown half off him, his untidy wig put on just as it happened [...] Something 
especially reckless in his demeanour, not only gave him a disreputable look, but so diminish the strong resemblance that he undoubtedly bore to the prisoner" (Dickens, 1981: 77). De esta forma, el narrador no sólo describe a Carton de manera que contrasta con la apariencia de Darnay, en particular con su forma de vestir y arreglarse el cabello, sino que también utiliza adjetivos que demuestran sus diferencias morales al describir a Carton como "reckless" y, sobre todo, "disreputable", reflejando la preocupación que los victorianos sentían por la apariencia y la reputación, estándares a los que Carton no se atiene. Como se aprecia en el pasaje, las diferencias entre sus personalidades son tan evidentes que incluso afectan la manera en la que se percibe su aspecto físico, de forma que aunque ambos sean aparentemente iguales, sus retratos los posicionan desde un inicio como opuestos.

Las diferencias entre ambos son resaltadas en su primera interacción, cuando conversan y cenan juntos y donde, por medio de sus discursos figurales, confirman los retratos de la voz narrativa. En esta conversación Carton le dice a Darnay: "This must be a strange night to you, standing alone with your counterpart on these street stones" (Dickens, 1981: 83). Aquí, él mismo se denomina la contraparte de Darnay, lo cual se enfatiza cuando Carton dice: "as to me, the greatest desire I have, is to forget that I belong to [this world]. It has no good on it for me-except wine like this-nor I for it. So we are not much alike in any particular, you and I" (Dickens, 1981: 84). Esto configura la caracterización de Carton como un hombre que no tiene mayores intereses en el mundo más que beber, restándole valores considerados positivos "at the heart of Victorian morality: self-control, self-help, self-reliance, self-discipline" (Madden, 1961: 99). A pesar de que Carton aparenta ser un personaje sin aspiraciones en su vida y quién podría no tener mayor peso en la trama de la novela, a partir de su aparición se crea "a tension between the role a character seems designed to play in a novel and some deeper purpose that character appears to serve" (Rosenberg, 1992: 149). Entonces, su apariencia física, similar en extremo a la de Darnay, no puede ser algo trivial o casual, sino que debe servir de alguna manera al desarrollo de la trama.

La caracterización de Darnay como un opuesto a Carton, en especial en su sentido del deber moral, se da en varios pasajes a lo largo de la novela. En éstos se demuestra que su moralidad va mucho más allá de lo reflejado en su retrato al mantener una apariencia y reputación respetable, pues también se preocupa por ejercer valores tales como la empatía y la solidaridad. El primer momento donde esto se puede ver es cuando renuncia por completo a ser parte de la aristocracia francesa al decirle a su tío, el Marqués Evrémonde: "we have done wrong, and are reaping the fruits of 
wrong [...] injuring every human creature who came between us and our pleasure" (Dickens, 1981: 125). Esta afirmación, dada por medio de su discurso figural, demuestra que está consciente de que su familia ha actuado de manera inmoral y de que él no está exento de la culpa, lo que se refleja al usar el "we". De igual manera, Darnay se desarrolla como un personaje honesto, lo cual se ve en su deseo de contarle al Doctor Manette su historia y verdadera identidad: "my present name [...] is not my own. I wish to tell you what that is, and why I am in England" (Dickens, 1981: 138). Por último, su caracterización, como alguien con "the glorious vision of doing good" (Dickens, 1981: 245), se ve por medio de su convicción de sacrificarse por alguien sólo porque es lo correcto, al decidir ir a Francia a salvar a Gabelle, sin tomar en cuenta lo que esto implica para él mismo. De esta forma, Darnay es retratado por sus acciones y su discurso como un hombre honesto, con un gran sentido de lo correcto y dispuesto a sacrificarse por los demás, lo cual contrasta con la caracterización de Carton como alguien sin propósitos en la vida.

A pesar de ser representados desde un inicio como opuestos, se comienza a desarrollar un cambio en Carton que se refleja a lo largo de la novela por medio de su discurso figural. Este cambio comienza desde una conversación que tiene con Lucie Manette, en donde le dice: "Since I know you [...] I have had unformed ideas of striving a fresh, beginning anew, and flighting out the abandoned fight..." (Dickens, 1981: 152). En este pasaje, Carton comienza a demostrar una inquietud por hacer un cambio en sí mismo y por volver a encontrarle un sentido a la vida. En esta conversación también es donde hace la promesa: "for you, and for any dear to you I would do anything" (Dickens, 1981: 152), la cual no sólo va a cumplir, sino que le da un propósito moral. A partir de este momento, Carton comienza a cambiar de manera gradual a través de pequeñas acciones como acercarse a Darnay y hacerse su amigo, o por el hecho de que "he never came heated with wine" (Dickens, 1981:213) desde el momento que Lucie y Charles tienen a su hija, lo cual comienza a demostrar su transformación.

Hacia el final de la novela, cuando Carton decide salvar a Charles Darnay, este cambio en él mismo es tan radical que la gente a su alrededor lo nota. Miss Pross reflexiona sobre él por medio de su discurso figural narrativizado: "there was a braced purpose in the arm and a kind of inspiration in the eyes which not only contradicted his light manner, but changed and raised the man" (Dickens, 1981: 303). Su cambio interno se empieza a notar a través de sus brazos y ojos, en donde se demuestra cómo los atributos morales se reflejan en los físicos. La otra 
persona en notar su cambio es nada menos que su supuesta contraparte, Darnay, cuando "the door was quickly opened and there stood before him, face to face, quiet, intent upon him, with the light of a smile in his features, and a cautionary finger on his lips, Sydney Carton. There was something so bright and remarkable in his look" (Dickens, 1981: 355). En este pasaje, donde se ven "face to face" Carton y Darnay, se revelan finalmente como espejo del otro de forma que Darnay puede ver reflejadas por fin sus propias características positivas en Carton. Sin embargo, el último indicador y más importante, es que Carton por fin ve por él mismo su cambio interior, lo que se refleja en la última frase de la novela, la cual forma parte de su discurso figural: "It is a far, far better thing that I do, than I have ever done..." (Dickens, 1981: 382). En ésta reconoce que fue capaz de hacer algo bueno aun cuando era algo que nunca había hecho o pensado hacer en su vida. Así, para el final de la novela, ambos personajes son valientes, determinados y dispuestos a sacrificarse por la gente que les importa, por lo que sus estándares morales van mucho más allá de las características de respetabilidad de la época.

$\mathrm{Al}$ principio de la novela parece haber dos extremos morales establecidos e inamovibles marcados de manera clara por la voz narrativa a través del contraste y la oposición. Por un lado, está el extremo positivo, que de acuerdo con la primera oración representa "light”, “wisdom” y "hope”, y por el otro lado está el extremo moral negativo que representa "darkness", "foolishness" y "despair". En los primeros capítulos de la novela, la voz narrativa caracteriza a los personajes a través de sus retratos de forma que encajan con los extremos positivos y negativos de acuerdo con los estándares morales de la época, sobre todo con respecto a su "respetabilidad". Se podría decir que por un lado se encuentran el Doctor Manette, Jarvis Lorry y Charles Darnay, que en sus retratos iniciales muestran valores como trabajo duro, mesura y sobriedad y quienes cuentan con buena reputación. Por el otro lado, la voz narrativa posiciona en el extremo contrario a Monsieur Defarge, Jerry Cruncher y Sydney Carton, ya que son personajes a quienes de distintas formas no les importa atenerse a los estándares de lo socialmente aceptable.

A medida que se desarrolla la novela y a través del discurso figural y de las acciones de los personajes, se matiza la manera en que estos extremos morales están configurados y se sugiere que éstos van mucho más allá de lo considerado "respetable". Esto se demuestra, en primera instancia, por medio de la capacidad de los tres personajes que la voz narrativa había retratado en el extremo moral negativo de evolucionar a lo largo de la historia hasta compartir características con sus supuestas contrapartes. 
El hecho de que los personajes que en un principio son posicionados en extremos distintos - $y$, en teoría, excluyentes - compartan varias similitudes demuestra que en realidad existe un espectro entre estos dos extremos donde estas similitudes convergen. Esto, asimismo, matiza la manera en la que se conceptualizan los extremos morales. Por ejemplo, se demuestra que el extremo moral "positivo" va mucho más allá de los estándares de respetabilidad y se conforma en su lugar por características como compasión, lealtad, valentía, la capacidad de formar relaciones significativas y, sobre todo, el potencial de transformación y crecimiento. Este aspecto contrasta con otros personajes presentes en la novela, tales como Madame Defarge, quien es guiada sobre todo por impulsos como la venganza y el odio. Debido a que Defarge está aferrada a estos impulsos, como consecuencia de su historia familiar y posición social, en el momento en el que interactúa con Lucie, su contraparte, no demuestra una capacidad para ser receptiva a ella o reformularse, por lo que no tiene la posibilidad de redimirse. La caracterización de Defarge también matiza el extremo moral negativo, pues demuestra que su postura se debe a circunstancias específicas y traumáticas en su vida. Sin embargo, el que no sea capaz de transformarse más allá de estos eventos le niega la capacidad de la movilidad entre los extremos morales.

En lo que respecta a los personajes que sí logran una transformación moral, a lo largo de la novela se da un contraste entre sus retratos iniciales provistos por la voz narrativa y el discurso figural de éstos. El discurso figural sirve como una forma de distanciarse en ciertos aspectos de esos retratos iniciales y marcar una evolución identitaria desde su propia voz y experiencia, pues como establece Pimentel (1998), "el discurso figural [funciona] como el vehículo privilegiado para marcar una postura frente al mundo" (94). La reformulación de la identidad por parte de los tres pares de personajes analizados en este trabajo demuestra cómo ésta no se trata de un constructo fijo, sino algo que está en constante cambio y que puede ser influenciado por el entorno y las personas con las se establece relación. Esto se demuestra en el hecho de que los personajes no cambian solos, ya que en algún punto todos interactuaron con su aparente "contraparte" y, por medio de las características de éstos, logran reformular su propia identidad de forma positiva. Como Gillman y Patten (1985) sugieren, "Doubles for Dickens become ways of expressing the spectrum of possibilities for character" (444), lo cual se refleja en el mismo discurso de Carton, quien establece que las interacciones con sus dobles demuestran, por una parte, "what you have fallen away from, and what you might have been" (Dickens, 1981: 85) y, al mismo tiempo, develan en lo que se pueden llegar a convertir. Así, se enfatiza que lo que 
tiene mayor importancia no es qué tan distintos seamos del otro, sino que siempre hay formas en las que podemos llegar a ser similares y que estas similitudes pueden ser más poderosas.

El hecho de que exista una escala de grises entre los extremos morales retratados en la que "héroes" y "villanos" comparten características provoca un cuestionamiento de la forma en la que estas categorías se construyen en primer lugar y sugiere, al mismo tiempo, que ninguno de los personajes es perfecto y que todos tienen oportunidad de cambiar. Esto se ve reflejado en la novela ya que los personajes que se podrían clasificar como "héroes" son los que tienen la capacidad de sobreponerse a los errores que han cometido y de reformularse de manera positiva, y no únicamente los personajes bien vistos por la sociedad. Por otro lado, también se demuestra que quienes podrían ser catalogados como "villanos" deben esta posición a circunstancias sociales y personales específicas y que, en la mayoría de los casos, éstas no impiden una reformulación de su identidad de forma positiva. La escala de grises creada hace que las categorías de "héroes" y "villanos" o "buenos" y "malos" sean inadecuadas para definir a los personajes, pues, a lo largo de la novela, se presentan más que nada como humanos interactuando con su entorno, tomando decisiones morales complejas y reformulando su identidad, lo cual, en la trama de la novela, cuenta en sí mismo con algo de heroísmo. Esto, a su vez, reconceptualiza la manera en la que se solía juzgar la moralidad y se lleva más allá de ciertos valores establecidos en específico por la clase media.

Así, Dickens logra representar la dualidad de forma ambivalente en A Tale of Two Cities al marcar una distinción clara por medio de los retratos dados por la voz narrativa entre los personajes que pertenecen en un inicio al extremo moral negativo y positivo, y, a la vez, demostrar a través de sus discursos figurales que éstos comparten características y que son capaces de modificar la manera en que se mueven dentro de este espectro. Con esto también se podrían sugerir similitudes entre otras dualidades representadas en la novela. Por ejemplo, aunque Francia e Inglaterra se representan como distintos ya que Francia se retrata como un espacio despiadado y violento por las circunstancias, también se sugiere que en Inglaterra existen tensiones sociales y actitudes similares entre las clases altas y las clases bajas que responden a situaciones morales y políticas complejas. Así, al proyectar valores y constructos ingleses victorianos a la Francia de la Revolución, puede que Dickens estuviera señalando que estas épocas corrían el riesgo de no ser tan diferentes una de la otra si Inglaterra no era cuidadosa al manejar estas tensiones. Al transmitir esto, Dickens nos recuerda 
que pueden y suelen existir similitudes entre nuestras naturalezas como humanos sin importar la clase social, el país o época en el que nos encontremos, lo cual desafía ciertas ideologías nacionalistas y de clase presentes en la época.

En el primer capítulo de la novela la voz narrativa comenta que las autoridades del periodo eran las que consideraban la época llena de contrastes, pero como Devin Griffiths (2013) establece, "if these authorities rely upon the 'superlative degree of comparison only', the narrator opens the possibility that less superlative degrees will tell a more accurate history" (812). Dickens utiliza "less superlative degrees" al relatar la Revolución Francesa a través de las vidas de individuos anónimos, pero no por eso menos heroicos, en la historia de la revolución, y por medio de éstos evidencia las posibilidades existentes entre los opuestos, las cuales hacen que la historia contada sea más humana. Es gracias a estos personajes que la novela sucede en realidad en "the best of times", "the worst of times", y en toda la escala de grises entre éstos.

\section{Referencias bibliográficas}

AL-Zuhairi, Ali Abdullah. (2016). "Dickens's Neutral Philosophy of the French Revolution in Tale of Two Cities". Journal of Language Teaching and Research, 7(6), 1123-1127. http://dx.doi.org/10.17507/jltr.0706.09

Davis, Paul. (2008) “A Tale of Two Cities". En David Paroissien (Ed.), A Companion to Charles Dickens (pp. 412-421). Blackwell.

Dennis, Carl. (1969). “Dickens' Moral Vision”. Texas Studies in Literature and Language, 11(3), 1237-1246.

Dickens, Charles. (1981 [1859]). A Tale of Two Cities. Bantman Classics.

Fludernik, Monika. (2007). "Identity/Alterity." En David Herman (Ed.), The Cambridge Companion to Narrative. Cambridge University Press.

Glancy, Ruth (Ed.). (2006). Charles Dickens's A Tale of Two Cities: A Sourcebook. Routledge.

Griffiths, Devin. (2013). “The Comparative History of a 'A Tale of Two Cities”. ELH, 80(3), 811-838.

Gillman, Susan K.; Patten, Robert L. (1985). “Dickens: Doubles: Twain: Twins”. Nineteenth-Century Fiction, 39(4), 441-458. https://doi.org/10.2307/3044714 
Golubov, Nattie. (2015). El circuito de los signos: una introducción a los estudios culturales. UNAM.

HutTer, Albert D. (1978). "Nation and Generation in A Tale of Two Cities." PMLA, 93(3), 448-462. https://doi.org/10.2307/461866

Madden, William A. (1961). "Victorian Morality: Ethics Not Mysterious". The Review of Politics, 23(4), 458-471.

Pimentel, Luz Aurora. (1998). El relato en perspectiva. UNAM; Siglo XXI.

Rosenberg, Brian. (1992). “Character and Contradiction in Dickens”. Nineteenth-Century Literature, 47(2), 145-163. https://doi.org/10.2307/2933634

Shires, Linda M. (2001). "The Aesthetics of the Victorian Novel: Form, Subjectivity, Ideology." En David Deidre (Ed.), The Cambridge Companion to the Victorian Novel. Cambridge University Press.

XIAO, Bin. (2015). "Morality in the Victorian Period." Theory and Practice in Language Studies, 5(9), 1815-1821. https://doi.org/10.17507/tpls.0509.07 